\title{
Gibt es Unterschiede in den gesundheitsschädlichen Wirkungen von Chrysotil- und Amphibol-Asbest?
}

\author{
Do Advers Health Effects of Chrysotile and Amphibole Asbestos Differ?
}

Autoren

Institute
X. Baur ${ }^{1}$, J. Schneider ${ }^{2}$, H.-J. Woitowitz ${ }^{2}$, M. Velasco Garrido ${ }^{1}$

Zentralinstitut für Arbeitsmedizin und Maritime Medizin (Direktor: Prof. Dr. med. Xaver Baur), Universitätsklinikum Hamburg-Eppendorf

2 Institut und Poliklinik für Arbeits- und Sozialmedizin (Komm. Leiter: Prof. Dr. med. Joachim Schneider), Universitätsklinikum Gießen und Marburg GmbH, Standort Gießen eingereicht 20.4.2012 akzeptiert nach Revision 12. 6. 2012

\section{Bibliografie}

DOI http://dx.doi.org/ 10.1055/s-0032-1309980 Pneumologie 2012; 66: 497-506 (c) Georg Thieme Verlag KG Stuttgart · New York ISSN 0934-8387

\section{Korrespondenzadresse} Prof. Dr. med. X. Baur Zentralinstitut für Arbeitsmedizin und Maritime Medizin, Universitätsklinikum Hamburg-Eppendorf Seewartenstraße 10 20459 Hamburg xaver.baur@bgv.hamburg.de

\section{Zusammenfassung $\nabla$}

Ausgehend von der aktuellen Infragestellung gesundheitsschädlicher Effekte des früher ganz vorrangig eingesetzten Chrysotil (Weiß-)-Asbests erfolgte eine Auswertung der Literatur über Invitro-, tierexperimentelle und epidemiologische Untersuchungen zur Wirkung der verschiedenen Asbestarten.

Chrysotil verursacht nach epidemiologischen Untersuchungen seltener Pleurafibrosen und Pleuramesotheliome als andere Asbestarten. Ansonsten zeigt Chrysotil keine eindeutigen Abweichungen im inflammatorischen, toxischen, kanzerogenen und Fibrose-induzierenden Potenzial im Vergleich zu anderen arbeitsanamnestisch relevanten Asbestarten. Diese Aussagen stützen sich auf klinische, tierexperimentelle und In-vitro-Befunde. Hieraus ergibt sich, dass die Negierung eines Ursachenzusammenhangs, z. B. bei Vorliegen einer Lungenfibrose (Asbestose) oder einer Erkrankung an Lungenkrebs nach einer Chrysotilbelastung von 25 Faserjahren und gleichzeitig weitgehendem Fehlen von Asbestfasern oder Asbestkörpern im Lungengewebe (s. sog. „Fahrerfluchtphänomen“), dem medizinisch-wissenschaftlichen Kenntnisstand widerspricht.

\section{Einführung}

\section{$\nabla$}

Die Deutsche Gesellschaft für Arbeitsmedizin und Umweltmedizin und die Deutsche Gesellschaft für Pneumologie und Beatmungsmedizin (DGP) haben in Zusammenarbeit mit der Deutschen Gesellschaft für Pathologie, der Deutschen Röntgengesellschaft und der Deutschen Gesellschaft für Hals-Nasen-Ohren-Heilkunde, Kopf- und Halschirurgie eine S2-Konsensus-Leitlinie zur Begutachtung durch Asbest verursachter Erkrankungen erarbeitet [1]. Wesentliche Beweggründe waren die hierzulande bis dato häufig sehr heterogene, nicht qualitätsgesicherte und oftmals nicht dem

\section{Abstract \\ $\nabla$}

Due to the current query whether the predominantly used chrysotile (white) asbestos comprises health risks we performed a literature search including in vitro and animal experiments as well as epidemiological studies.

As shown by epidemiological studies chrysotile causes less pleural fibrosis and mesotheliomas when compared with other asbestos types. However, its otherwise inflammatory, toxic, carcinogenic, and fibrosis-inducing effects correspond to those of other occupationally relevant asbestos types. This is based on clinical, animal as well as on in-vitro findings. This means that denying a causal relationship, e.g. in a case with lung fibrosis (=asbestosis) or lung cancer with an asbestos load of 25 fiber-years in the absence of identification of a significant concentration of asbestos fibers or asbestos bodies in the lung (see so-called "hit and run" phenomenon), contradicts the medical-scientific knowledge.

aktuellen medizinisch-wissenschaftlichen Kenntnisstand entsprechende Diagnostik, Begutachtung und Entschädigungspraxis asbestbedingter Erkrankungen.

In dieser Leitlinie erfolgte keine Differenzierung hinsichtlich der verschiedenen Asbestarten. Aufgrund der geringeren Biobeständigkeit des Chrysotilasbestes [2] werden jedoch mögliche Unterschiede der pathogenen Wirkung verschiedener Asbestarten weiterhin kontrovers diskutiert.

Bereits in den 1970er- und 1980er-Jahren wiesen mehrere Arbeitsgruppen darauf hin, dass der in Deutschland zu etwa 94\% verwendete ChrysotilAsbest im Lungengewebe von Patienten mit 
asbestbedingten Lungenerkrankungen nur sehr wenige Prozent der dort auffindbaren Asbestfasern ausmacht, während Amphibol-Asbestarten, zumeist Blauasbest (Krokydolith), Anthophyllit und Braunasbest (Amosit), welche nur 6\% des Asbestverbrauchs in Deutschland umfassten, im Lungengewebe dominieren [3-7]. Die Aufspleißung des Chrysotils in Elementarfibrillen [8, 9], die Magnesium-Auswaschung und die tierexperimentell gewonnenen Halbwertzeiten von nur wenigen Wochen [2] liefern die Erklärungen für dieses sog. „Fahrerfluchtphänomen“.

Die S2k-Leitlinie fasst diese Beobachtungen in folgenden Kernaussagen zusammen:

„Wichtig für die Beurteilung der Ergebnisse der lichtmikroskopischen als auch der elektronenmikroskopischen Analysen ist, dass in der Regel keine erhöhten Chrysotilfaserkonzentrationen im Lungengewebe nachgewiesen werden und damit die Aussagen beider Methoden hinsichtlich der stattgehabten Chrysotilexposition eindeutig limitiert sind (Lichtmikroskopie limitiert wegen Fasergröße, Elektronenmikroskopie wegen des sog. Fahrerfluchtphänomens).“ [10]

„Staubanalytische Untersuchungen (Lungengewebe/BAL) können die Feststellungen aus der Arbeitsanamnese und aus den Ermittlungen der TAD(Anmerkung: Technische Aufsichtsdienste der Unfallversicherung) nicht ersetzen und nicht Anlass sein, die ermittelte kumulative Exposition nach unten zu korrigieren. Sie sind aber geeignet, um weitere Ermittlungen zu bislang unbekannten Expositionsquellen auszulösen. Staubanalytische Untersuchungen von Lungengewebe oder einer BAL ersetzen keinesfalls die qualifizierte Arbeitsanamnese oder die Ermittlungen der Präventionsabteilungen.“ [11]

„Es gibt keinen staubanalytischen Grenzwert für asbestbedingte Lungenveränderungen“. [12]

Obwohl die im Konsens verabschiedete wissenschaftliche Leitlinie demzufolge von annähernd gleichartigen Wirkungen aller Asbestarten ausgeht, werden in der Praxis diametral entgegengesetzte Beurteilungen zu Lasten der Patienten beobachtet: Im berufsgenossenschaftlichen Deutschen Mesotheliomregister [1317] werden im Auftrag der gewerblichen Berufsgenossenschaften Lungengewebe auf ihren Gehalt an Asbestkörpern im Rahmen von tausenden Berufskrankheiten-Feststellungsverfahren obergutachtlich untersucht. Als zentrale Anlaufstelle der ermittelnden Verwaltungen für Staubanalysen kommt diesem Mesotheliomregister bisher eine Art Monopolstellung zu [17,18], obwohl in der Falkensteiner Empfehlung der DGUV [19], die sich an die v. g. Leitlinie anlehnt, auch andere Untersuchungseinrichtungen genannt werden, die ggf. zu Lasten der Unfallversicherung herangezogen werden können. Es wird angeführt, dass bei fehlendem Nachweis einer erhöhten Anzahl von Asbestkörpern bzw. -Fasern sich pathologisch-anatomisch keine Hinweise auf eine vergleichsweise vermehrte Asbestbelastung ergeben sollen. Daraus wird gefolgert, dass in solchen Fällen eine Berufskrankheit nicht vorliegt. Dies steht teilweise im Gegensatz zu sicherheitstechnischen Expertisen mit Bestätigung einer eindeutig erhöhten Asbestfaserstaubbelastung am Arbeitsplatz. Demzufolge wird nicht selten auf Basis von Asbestkörper- oder Faserzählungen im Gegensatz zur vorgenannten Leitlinie sowie zu arbeitsmedizinischen und pneumologischen Zusammenhangsgutachten das Vorliegen einer Berufskrankheit verneint (vgl. Fallbeispiel auf der folgenden Seite). Neumann et al. [20] gehen davon aus, dass Chrysotil aufgrund seiner geringen Biobeständigkeit kaum fibrogene Wirkung hat und aufgrund der „Fahrerflucht“ ein Schaden in Form einer Lungenfibrose nicht unterstellt werden kann. Auch würden Chrysotilfasern nicht zur Pleura gelangen.
Weltweit gibt es seit langem Diskussionen, ob die einzelnen Asbestarten unterschiedliche Gesundheitsrisiken aufweisen [21, 22]. Vor allem von den Protagonisten der kanadischen Asbestindustrie wird in jüngster Zeit vermehrt eine „sichere“ Chrysotil-Anwendung propagiert [23]. Trotz internationaler öffentlicher Proteste (der regionale Ärzteverband Quebecs vermied bemerkenswerterweise eine Stellungnahme hierzu) wurde eine neue Chrysotil-Mine mit staatlicher Unterstützung in Kanada eröffnet [24].

In dieser Arbeit sollen die Ergebnisse epidemiologischer, tierexperimenteller und In-vitro-Untersuchungen zur Frage potenzieller Unterschiede in den gesundheitsschädlichen Effekten verschiedener Asbestfaserarten dargelegt werden. Datengrundlage sind systematische Übersichten zu epidemiologischen Arbeiten, tierexperimentellen und In-vitro-Studien. Die Ergebnisse der berücksichtigten Studien werden narrativ dargestellt.

\section{Ergebnisse}

\section{Epidemiologische Untersuchungen \\ Lungenkrebs}

Lungenkrebs wird von allen Asbestarten hervorgerufen [25 - 29]. In ihrer Metaanalyse von 15 Studien fanden Berman und Crump [30] allerdings einen signifikanten Unterschied des LungenkrebsPotenzials von Chrysotil- und Amphibol-Asbest mit dünnen Fasern (Durchmesser $<0,4$ und $<0,2 \mu \mathrm{m}$ ), der für dickere Fasern nicht festzustellen war.

In einer aktuellen Metaanalyse führten Lenters et al. [31] eine Bewertung der Qualität der Expositionsbeurteilung in den einzelnen Studien anhand von fünf Aspekten durch (Dokumentation der Exposition; Expositions-Ratio zwischen höchster und niedrigster Expositionskategorie; Umrechnungsfaktor für verschiedene Faserbestimmungsmethoden; Anteil der durch Messung bzw. durch Abschätzung erfassten Expositionhöhe; Detailtiefe der Erfassung der Arbeitsanamnese). Nicht alle eingeschlossenen Studien hatten für Rauchen adjustiert. Aus Dosis-Wirkungs-Analysen der einzelnen Publikationen ermittelten die Autoren die Kanzerogenitäts-Stärke (sog. $\mathrm{K}_{\mathrm{L}}$-Wert) mittels linearer Regression. Der $\mathrm{K}_{\mathrm{L}^{-}}$ Wert stellt den Anstieg des Relativen Risikos pro Einheit der kumulativen Asbestexposition dar, je größer $\mathrm{K}_{\mathrm{L}}$, desto größer die kanzerogene Potenz. Anschließend berechneten sie den Gesamtschätzer der Kanzerogenität für alle Studien (Meta- $\mathrm{K}_{\mathrm{L}}$ ). Die Metaanalyse zeigte einen hohen Grad an Heterogenität $\left(1^{2}=64 \%\right)$. Die Stratifikation nach Asbesttyp ergab ca. $8 \times$ stärkere Effekte von Amphibol-Asbest als von Chrysotil-Asbest (Meta- $\mathrm{K}_{\mathrm{L}}$-Werte: Amphibole 0,33 [95\% CI 0,09; 0,56]; Chrysotil 0,04 [95\% CI-0,05, 0,12]). Die Stratifikation der Ergebnisse nach Expositionsbeurteilungs-Charakteristika belegte, dass Studien mit gut dokumentierter Expositionsbeurteilung, umfangreicherem Expositionsvergleich, genauerer Erfassung der Expositions-Vorgeschichte durch Messdaten und vollständigeren Arbeits-Vorgeschichten stärkere Effekte aufwiesen als Studien, die diese Merkmale nicht erfüllten. Es besteht also ein Zusammenhang zwischen Qualität der Expositionserfassung und Kanzerogenitätstärke. Es gab nur 2 der insgesamt 19 eingeschlossenen Arbeiten, die alle fünf oben genannten Qualitätsmerkmale erfüllten. Würde man nur diese beiden Studien betrachten, wäre die Kanzerogenität von Chrysotil ca. $8 \times$ stärker als die von Amphibol-Asbest [ $\mathrm{K}_{\mathrm{L}}$ Chrysotil 1,64 (95\% CI 0,80, 2,49); $K_{L}$ Amphibole 0,23 (95\% CI-0,20; 0,65)]. Gerade in den Studien der Chrysotil-Kohorten deutet sich an, dass mit Zunahme der Qualität der Expositionsbeurteilung die ermit- 


\section{Fallbeispiel}

Mann, 64 Jahre alt.

\section{Berufsanamnese:}

1963-1966 Lehre: Hitze- und Brandschutzplatten beim Schweißen verwendet, Wellasbestplatten geflext, Lüftungskanäle mit Spritzasbest ausgespritzt. In Zeiten, in denen er nicht selbst spritzte, bestand eine Bystander-Exposition. Helfer im Heizungsbau, dabei Arbeiten mit Spritzasbest.

1966 - 1973 Technischer Zeichner (Fachrichtung Heizungs-, Klima- und Sanitärtechnik).

1973 - 1977 Maschinenbaustudium und Schweißtechnik.

1987-2008 Prüfingenieur von Tank-, Dampf- und Druckanlagen, im Heizungsbau Asbestverkleidungen entfernt.

TAD: Asbestbelastung mindestens 25 Faserjahre.

\section{Klinische Anamnese:}

Seit Jahren progrediente Dyspnoe, 01/2008 Lungenbiopsie mit histologischer Sicherung einer Fibrose (UIP-Muster), sekundär pulmonale Hypertonie mit seit 2010 mittelgradiger respiratorischer Insuffizienz. Aktuell Lungentransplantationsvorbereitung.

\section{Befunde:}

Auskultation: Knisterrasseln über beiden basalen Lungenfeldern.

Lungenfunktion: VK 54\% und $\mathrm{DL}_{\mathrm{CO}-\mathrm{SB}} 31,5 \%$ des Sollmittelwertes

\section{HRCT: Wabenlunge, einzelne Kalkstippchen in der Pleura}

Histologie der explantierten Lunge: ausgedehnte subpleural bzw. peripher betonte Fibrose und Vernarbung des Lungenparenchyms immer wieder unter Einschluss anthrakosilikotischer Mischstaubablagerungen, teils wabiger Umbau des Lungenparenchyms mit stellenweise dystrophen Verkalkungen. Pleura verfestigt und weißlich, im Unterlappen eine weiße Plaque-artige Struktur.

\section{Pathologische Stellungnahme}

Es wird erwähnt, dass nach Angaben der Unfallversicherung eine Belastung von mindestens 25 Asbestfaserjahren bestand. Der Patient litt an einer fortgeschrittenen Lungenfibrose („bis hin zur initialen Wabenlunge“) vom Typ einer UIP. 0,2g Lungengewebe waren für die sog. „Lungenstaubanalyse“ verfügbar. Hochgerechnet auf $1 \mathrm{~g}$ ergaben sich „wenn überhaupt, weniger als 8 Asbestkörper, in der ersten Probe aber 44 und in der zweiten Probe 7 Substanzen mit nicht identifizierbarer Zentralfaser.“ Weiter heißt es: „Asbest-assoziierte fibrosierende Lungenveränderungen vom Typ der Asbestose oder Minimalasbestose konnten lichtmikroskopisch ausgeschlossen werden. Nach dem Ergebnis der Lungenstaubanalyse ist auch nicht davon auszugehen, dass dem Asbest die Wertigkeit einer Ursache oder wesentlichen Teilursache für die Entstehung und Unterhaltung der fibrosierenden Lungenveränderungen zukommt.“

Kommentar: Zunächst verwundert die winzige, zur „Faserstaubanalyse“ eingesetzte Gewebsprobe aus der explantierten Lunge. Die vorgenannte, einer Fachbegutachtung entsprechende Stellungnahme ignoriert das in der Einleitung angeführte, vielfach belegte Fahrerfluchtphänomen des Chrysotils und die damit verbundene Präferenz der Arbeitsanamnese und sicherheitstechnischen Dosisabschätzung gegenüber der Faser- bzw. Asbestkörper-Quantifizierung im Lungengewebe. Hinzu kommt, dass in den winzigen Gewebeproben 44 bzw. 7 „faserförmige Substanzen“ nicht identifiziert wurden, also angesichts der Anamnese möglicherweise Asbestfasern sind. Der Anspruch erscheint außerordentlich fragwürdig, aus 0,2 g Lungengewebe, d.h. einer 0,2g Stichprobe - also dem ca. 0,00025sten Teil der ca. $800 \mathrm{~g}$ schweren Patientenlunge - die arbeitsanamnestisch und sicherheitstechnisch unstrittige massive Asbestgefährdung negieren zu können. Warum aus der explantierten Lunge nur derart winzige Proben für die „Faserstaubanalyse" eingesetzt wurden, bleibt unklar. Auch werden die Heterogenität der pulmonalen Asbestfaserablagerungen und die Variabilität mineralogischer Befunde nicht kritisch von Seiten der Fachgutachter gewürdigt.

Einen andernorts weiter zu prüfenden Nebenaspekt wirft die inzwischen von dem Patienten dem zuständigen Unfallversicherungsträger zugestellte Beschwerde auf, dass diese pathologische Untersuchung (in üblicher Weise) ohne Zustimmung des Betroffenen erfolgte.

\begin{tabular}{|c|c|c|c|c|c|}
\hline Studie/Autor & Fasertyp & $\begin{array}{l}\text { Erfüllte Qualitäts- } \\
\text { merkmale }\left[K_{L}\right]\end{array}$ & Stärke & $95 \% \mathrm{Cl}$ & \multirow{10}{*}{$\begin{array}{l}\text { Tab. } 1 \text { Kanzerogenitätsschätzer } \\
\left(\mathrm{K}_{\mathrm{L}}\right) \text { in Kohorten mit verschiede- } \\
\text { nen Asbestexpositionen und } \\
\text { unterschiedlicher Qualität der } \\
\text { Expositionserfassung. } 1=\text { niedrig, } \\
5=\text { hoch, Quelle: eigene Darstel- } \\
\text { lung nach Lenters et al. [31]. }\end{array}$} \\
\hline S. Carolina, USA [36] & Chrysotil & 5 & 1,64 & 0,$80 ; 2,49$ & \\
\hline N. Carolina, USA [37] & Chrysotil & 3 & 0,12 & $-0,03 ; 0,28$ & \\
\hline Quebec, CDN [38] & Chrysotil & 3 & 0,03 & 0,$02 ; 0,04$ & \\
\hline Balangero, I [39] & Chrysotil & 1 & 0,07 & $-0,11 ; 0,25$ & \\
\hline Connecticut, USA [40] & Chrysotil & 1 & $-0,15$ & $-0,33 ; 0,02$ & \\
\hline Libby, USA [41] & Amphibol & 5 & 0,23 & $-0,20 ; 0,65$ & \\
\hline Wittenoom, AUS [42] & Amphibol & 3 & 0,40 & 0,$05 ; 0,74$ & \\
\hline Tyler, USA [43] & Amphibol & 3 & 0,13 & $-0,23 ; 0,65$ & \\
\hline Paterson, USA [44] & Amphibol & 2 & 1,06 & 0,$34 ; 1,78$ & \\
\hline
\end{tabular}

telte Kanzerogenität $\left(\mathrm{K}_{\mathrm{L}}\right)$ stärker ist. Umgekehrt deuten sich unter den 4 Amphibol-Kohorten schwächere Effekte bei besserer Qualität der Expositionsbewertung an (siehe Tab.1).

Zusammenfassend ist dieser Metaanalyse zu entnehmen, dass Unterschiede bei Chrysotil- im Vergleich zu Amphibol-Asbestexponierten kaum festzustellen sind, wenn die Metaanalyse auf Studien mit weniger Schwächen in der Expositionsbewertung begrenzt wird. Es gibt nur sehr wenige Studien, die eine fundier- te, auf einer zuverlässigen Beurteilung der Exposition basierende Untersuchung der Unterschiede der Kanzerogenitätsstärke zwischen beiden Asbestformen erlauben. Die Metaanalyse zeigt die Limitationen der vorliegenden Arbeiten für die Untersuchung von Unterschieden in der Kanzerogenitätsstärke der verschiedenen Asbestformen und stellt die Annahme einer epidemiologischen Evidenz hinsichtlich eines Unterschieds des Lungenkrebsrisikos zwischen den verschiedenen Asbestarten in Frage. 
In einer soeben erschienenen prospektiven Kohortenstudie mit einem Follow-up von 37 Jahren [32, 33] wurde unter ChrysotilArbeitern einer chinesischen Textilfabrik eine signifikante DosisWirkungs-Beziehung bezüglich der Mortalität an Lungenkrebs festgestellt, wobei sich vergleichbare Korrelationsfaktoren wie für Amphibol-Asbest ermitteln ließen. Die Studie berücksichtigte in der Analyse unter anderem das Rauchen.

In einer weiteren Kohorte von chinesischen Arbeitern einer Chrysotil-Mine wurde eine 4-mal höhere Lungenkrebsmortalität bei den Bergleuten im Vergleich zu dem Verwaltungspersonal [34] festgestellt.

Elliott et al. [35] beschrieben kürzlich unter US-amerikanischen Chrysotil-exponierten Textilarbeitern ebenfalls signifikant erhöhte Lungenkrebsraten; nicht geklärt werden konnten dabei bisher deutliche Unterschiede zwischen den Standorten der Betriebe (Nord- und Süd-Carolina).

\section{Mesotheliom}

Das Mesotheliom-Risiko von Chrysotil-Asbest ist im Vergleich zu Amphibol-Asbest deutlich geringer ausgeprägt [29,45-50]. Hodgson und Darnton [51] haben in ihrer Analyse große Unterschiede im kanzerogenen Potenzial, im Sinne des Mesotheliomrisikos, das mit einem Faserjahr zusammenhängt, zwischen den verschiedenen Asbesttypen ermittelt, wobei Krokydolith 54-mal und Amosit 14-mal stärker kanzerogen war als Chrysotil [51]. Berman und Crump [30] berichteten über ähnliche Unterschiede im kanzerogenen Potenzial zwischen den verschiedenen Faserarten. Stayner et al. [22] berechneten, dass bei den 12 Kohorten mit beruflicher Exposition gegenüber Chrysotil-Asbest insgesamt $0,3 \%$ der Todesfälle auf ein Mesotheliom zurückzuführen waren, während der diesbezügliche Anteil in der allgemeinen Bevölkerung nur $0,02 \%$ betrug [22]. Eine aktuelle Übersichtsarbeit identifizierte insgesamt 30 Berichte über Kohorten ( $n=14$ Studien), Fall-Kontroll-Studien $(n=3)$, Fallserien und -berichte $(n=$ 13), in denen Chrysotil-Asbest die einzige oder die ganz überwiegende Exposition war [52]. In all diesen Studien wurden Mesotheliomfälle dokumentiert.

Ergebnisse einiger Studien zur Faserbelastung suggerieren, dass Chrysotil- und auch andere Asbestfasern insbesondere zur Pleura wandern, sodass Faserquantifizierungen im Lungengewebe nicht zuverlässig der Faserkonzentration im Pleuragewebe entsprechen $[22,53,54]$. In den toxikologisch-arbeitsmedizinischen Begründungen von MAK-Werten [26] findet sich über Asbest-Faserstaub folgende Feststellung:

„Insgesamt lassen sich aus den 3 Blickrichtungen

- der physiko-chemischen Eigenschaften (Verstaubungsverhalten, Faserdurchmesser, Löslichkeitsverhalten),

- der Untersuchungen an menschlichen Lungenstäuben mittels Elektronenmikroskopie sowie

- von epidemiologischen Untersuchungen durch verschiedene Arbeitsgruppen

Hinweise auf ein höheres Mesotheliom-erzeugendes Potenzial des Krokydolith im Vergleich zum Chrysotil gewinnen.“

Bemerkenswert ist die in Deutschland im Vergleich zu anderen Ländern, z.B. England, um ca. 50\% niedrigere Mesotheliomzahl (http://www.hse.gov.uk/statistics/), wobei nicht von wesentlichen Abweichungen der verbrauchten Mengen verschiedener Asbestarten auszugehen ist.

\section{Andere Tumoren}

Die Häufigkeit des Larynxkrebs ist sowohl unter Amphibol- als auch Chrysotil-Exponierten signifikant erhöht, wobei Hinweise auf positive Dosis-Wirkungs-Beziehungen bestehen [50, 55 - 57]. Laut der IARC [58] liegt eine ausreichende Evidenz für eine kausale Assoziation zwischen Asbest-Exposition und Ovarialkarzinom vor. Camargo et al. [59] fanden in einer Metaanalyse für das Ovarial-Karzinom folgende gepoolte SMRs (95\% CI) unter Asbestexponierten Frauen: Chrysotil 1,4 (0,88-2,21), Krokydolith 2,18 $(1,4-3,37)$, gemischte oder andere Asbestarten $2(1,41-2,84)$. Auch über eine Überhäufigkeit von Pharynx- und gastrointestinalen Tumoren, z. T. auch des Non-Hodgkin-Lymphoms, wird berichtet [57, 59 - 62], wobei hierbei keine Konsistenz der Ergebnisse besteht [33] und auch keine eindeutige Differenzierung nach Asbestarten möglich war.

\section{Asbestose und Pleuraplaques}

In einer kürzlich erschienenen Veröffentlichung wird postuliert, dass Chrysotil-Asbestfasern nicht zur Pleura gelangen [20]. Dabei wird völlig übersehen, dass gerade auch in der Bundesrepublik seit mehr als 40 Jahren gezielte epidemiologische Untersuchungen zu den Auswirkungen von Pleuraverkalkungen auf die Lungenfunktion speziell bei Chrysotil-Asbestarbeitern vorliegen [63]. Stayner et al. [22] konnten dann in ihrer Übersicht von 1996 mehrere Untersuchungen identifizieren, in denen eine Dominanz kurzer Chrysotilfasern in der Pleura, in Pleuraplaques und in fibrotischem Pleuragewebe festzustellen war. Gleichzeitig fand sich eine Dominanz von Amphibolfasern im Lungengewebe [22]. Bisher wurden Faserbelastung und Fasertyp bei Patienten mit Pleuraerkrankungen nur vereinzelt untersucht, wobei Pleuraplaques in Zusammenhang mit langen Amphibol- und kurzen Chrysotilfasern festzustellen waren [64].

In einer aktuell veröffentlichten HRCT-Untersuchung wurde bei Automobil-Mechanikern, die gegenüber Chrysotil-haltigen Stäuben von Bremsbelägen und von Kupplungen exponiert waren und sonst keinen weiteren Asbest-Kontakt hatten, eine Prävalenz von Pleuraplaques von 4,9\% und von diffuser Pleuraverdickung von 3,9\% beobachtet [65]. Darüber hinaus erwähnen die Autoren in dieser Studie unter Berücksichtigung der Anamnese einen Fall einer asbestbedingten Lungenfibrose.

Zusammenfassend finden sich auch unter Chrysotil-Exponierten Pleurafibrosen, z. T. verkalkt, wenngleich etwas seltener als unter Krokydolith- oder Mischasbest-Exponierten [25,66,67]. Diese Pleuraveränderungen zeigen keine eindeutige Dosisabhängigkeit; sie sind eher mit der Latenzzeit assoziiert [67 - 70].

In zwei Übersichtsarbeiten [61,71] wurden epidemiologische Studien zu Dosis-Wirkungs-Beziehungen von Chrysotil ausgewertet. Es zeigte sich, dass Asbestose-Erkrankungen hierbei häufig (bis zu 20\%) nach anhaltender Exposition gegenüber einer Konzentration von 5-20 Millionen Fasern $/ \mathrm{m}^{3}$ entsprechend einer kumulativen Belastung von 50-200 Millionen Fasern $/ \mathrm{m}^{3}$ über 10 Jahre auftreten. Das Follow-up-Update der South Carolina Chrysotil-Kohorte (Arbeiterinnen und Arbeiter einer Textilfabrik, bei der nahezu ausschließlich Chrysotil verwendet wurde) bestätigt den starken, dosisabhängigen Zusammenhang zwischen Chrysotil-Exposition und asbestosebedingter Mortalität [36].

Auch Deng et al. [32] konnten kürzlich unter Chrysotil-exponierten Textilarbeitern eine signifikante Dosis-Wirkungs-Beziehung für die Mortalität in Folge einer Asbestose feststellen. Insgesamt wurden ca. 15\% der Todesfälle in dieser Kohorte auf eine Asbestose bzw. nicht-maligne (asbestbedingte) respiratorische Erkran- 
kungen zurückgeführt (5-6-fache Erhöhung gegenüber nichtexponierten Kontrollen).

Bereits die frühen Auswertungen von Lungenfunktionsmessungen von Becklake et al. [67] ergaben unter Chrysotil-Asbestarbeitern restriktive Einschränkungen im Sinne von Dosis-Wirkungs-Beziehungen. Dies bestätigt sich in neueren Arbeiten $[72,73]$.

\section{Tierexperimentelle Daten}

Über die Prüfung von Asbest auf fibrogene und kanzerogene Wirkungen in Tierversuchen mit unterschiedlichen Verabreichungsarten liegt umfangreiche Literatur vor [71, 74-77]. In Inhalationsversuchen, vor allem an Ratten, entfalten sowohl Amphibolasbeste als auch Chrysotil qualitativ die beim Menschen bekannten Wirkungen auf die Lunge: Lungenfibrose, Lungentumoren, maligne Mesotheliome. In einer Reihe von Studien zeigten dabei Amphibolfasern ebenso wie Chrysotilfasern - bezogen auf die Faseranzahl-Konzentration in der Atemluft - auch quantitativ sehr ähnliche Wirkungsstärken, d.h. Relationen von Tumorhäufigkeit und Expositionskonzentration ( $\bullet$ Abb. 1). Nach einer Zusammenstellung von Daten der Arbeitsgruppe von Davis durch Pott et al. [78] waren die induzierten Tumorrisiken durch den Amphibol-Asbest Amosit und durch Chrysotil trotz geringerer in der Lunge retinierter Staubmasse ähnlich - bei ähnlichen Expositionskonzentrationen der Anzahl an Fasern mit Längen größer als $5 \mu \mathrm{m}$. Für Krokydolith war in dieser Versuchsreihe, entsprechend den niedrigeren Faseranzahl-Konzentrationen, das Tumorrisiko deutlich niedriger - trotz höherer retinierter Staubmasse in der Lunge ( $\bullet$ Abb.1). In anderen Studien traten zwar ähnliche Tumorhäufigkeiten nach Krokydolith- und Chrysotilexposition bei deutlich höheren Faseranzahlwerten von Chrysotil auf, so-

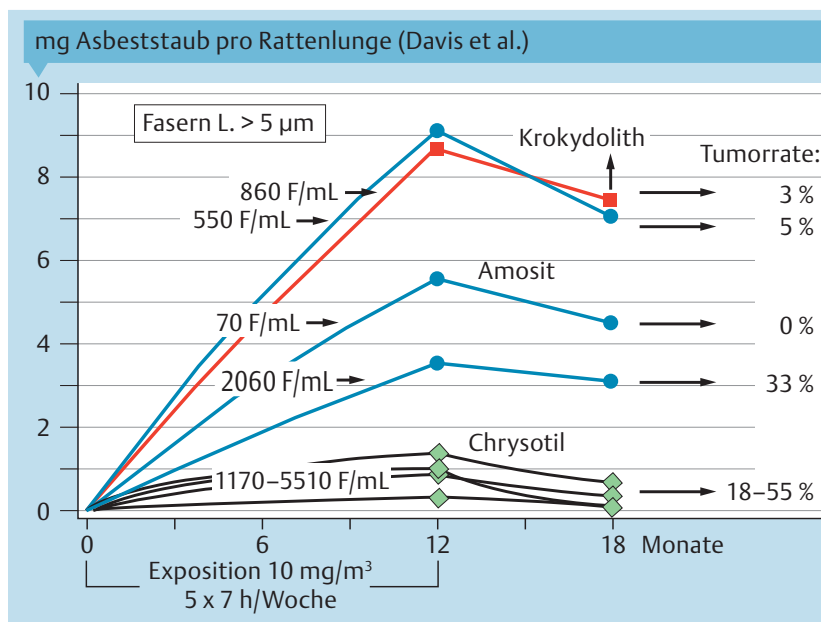

Abb. 1 Staubmasse pro Rattenlunge in Inhalationsversuchen der Arbeitsgruppe von Davis [80 - 84]. Die Darstellung von Pott, Roller et al. [78] veranschaulicht die im Vergleich zu Amosit und Krokydolith geringe Retention der Chrysotil-Staubmasse, die dennoch mit einer hohen Tumorhäufigkeit assoziiert ist. Die Kurven beschreiben den zeitlichen Verlauf der Staubmasse nach dem „konventionellen“ Modell (Invasion 0. Ordnung, Elimination 1. Ordnung). Hierzu wurde die Zeitkonstante aus den beiden Messpunkten bei 12 und 18 Monaten berechnet [78]. F=Fasern, L. = Länge.

dass rechnerisch eine geringere Wirkungsstärke des Chrysotils im Vergleich mit dem Krokydolith erscheint; die StaubmasseKonzentrationen in der Atemluft waren jedoch genau gleich (৫ Tab.2). Bezogen auf die Staubmasse-Konzentration ist daher auch in dieser Versuchsreihe eine ungefähr gleiche Wirkungsstärke der Chrysotil- und Krokydolithstäube in Bezug auf fibro-

Tab.2 Exposition, Faserzahl in der getrockneten Lunge, Tumorhäufigkeit (Lunge, Mesotheliom) und Fibrosegrad nach Inhalation von Krokydolith und Chrysotil in den RCC-Laboratorien, Genf, bei männlichen F344 / N-Ratten (Zusammenstellung aus Pott et al. [94] bzw. Roller [75]).

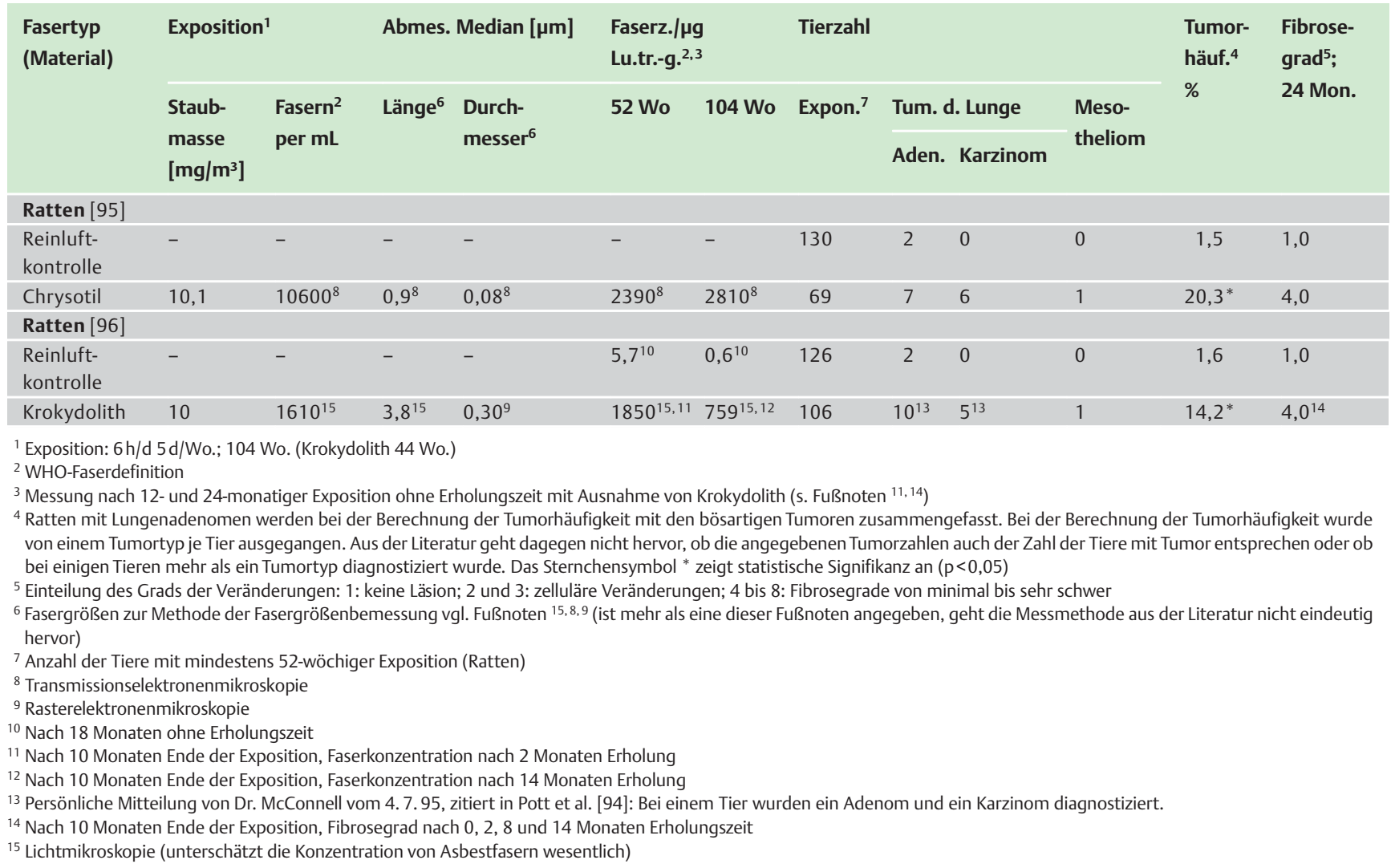




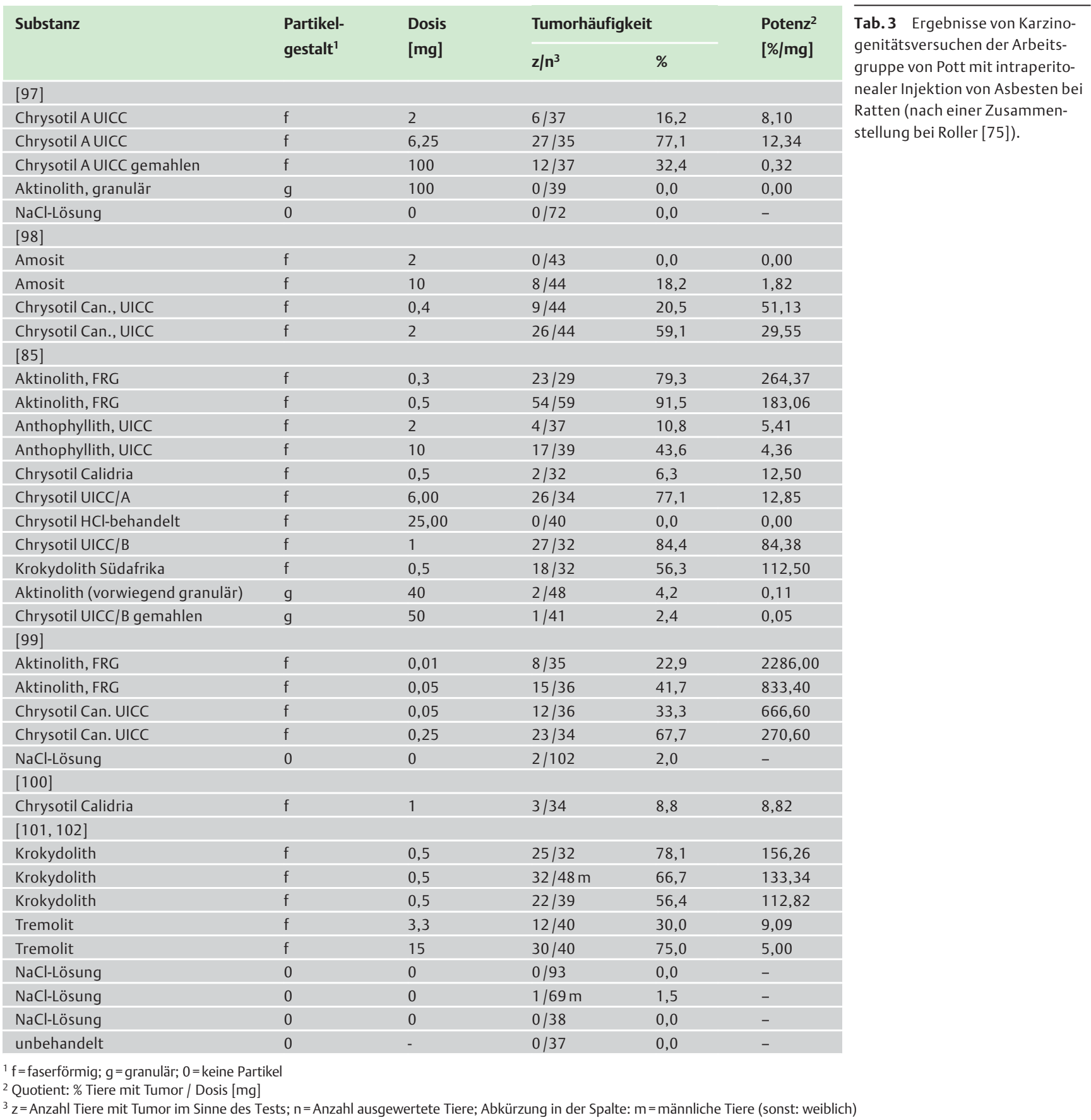

gene und kanzerogene Wirkung festzustellen; grundsätzlich sind dabei stets auch die Methoden der Fasermessung zu berücksichtigen (Lichtmikroskopie, Raster- versus Transmissionselektronenmikroskopie).

Sehr umfangreiche Daten liegen aus Versuchen mit intraperitonealer Injektion faserförmiger Stäube bei Ratten vor. $\bullet$ Tab. 3 zeigt eine Auswahl der Ergebnisse der Arbeitsgruppe von Pott mit verschiedenen Asbestvarianten, zum Teil auch gemahlenen (Zerstörung der Faserstruktur) und nicht-faserförmigen Aktinolithvarietäten. Für einen Vergleich der Wirkungsstärken sind insbesondere die niedrigeren Dosierungen geeignet, deshalb ist die Auswahl der Versuchsgruppen in $\bullet$ Tab. 3 auf die niedrigeren Dosierungen - der Faserproben - begrenzt (bei gemahlenen oder granulären Vergleichsproben wurden höhere Dosen verwendet). Tab. 3 veranschaulicht, dass es sowohl zwischen ver- schiedenen Proben von Amphibol-Asbesten als auch zwischen Amphibolen und Chrysotil, bezogen auf die verabreichte Staubmasse, unterschiedliche Wirkungsstärken (kanzerogene Potenz) gab, dass aber sowohl Amphibole als auch Chrysotil sehr hohe kanzerogene Wirkungsstärken auf die Serosa entfalten können. Die höchste Wirkungsstärke wurde von Pott et al. [79] mit einer Aktinolithprobe beobachtet, die mit nur $10 \mu$ g bei $23 \%$ der injizierten Tiere maligne Tumoren (Mesotheliome, Sarkome) induzierte. Eine Probe von kanadischem Chrysotil UICC/B stand dem kaum nach, $50 \mu \mathrm{g}$ induzierten bei 33\% der Tiere solche Tumoren. Krokydolith- und Tremolitproben sowie eine Probe von Chrysotil „Calidria“ erschienen, bezogen auf die Staubmasse, schwächer wirksam. Bei der Beurteilung der Wirkungsstärken, bezogen auf die Staubmasse, ist zu berücksichtigen, dass sich die Faseranzahl je mg Staub und deren Größenverteilungen unterscheiden; rät- 


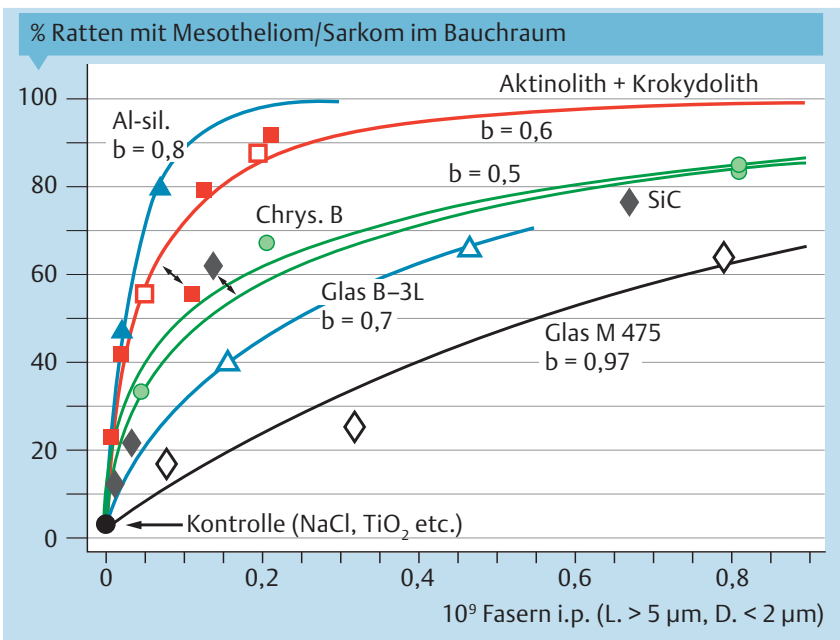

Abb.2 Beziehungen zwischen Dosis und Tumorhäufigkeit nach intraperitonealer Injektion faserförmiger Stäube (Daten von Pott et al. $[79,85,86])$. Die Kurven wurden durch eine Anpassung mit dem Weibull-Modell berechnet; dieses Modell ist eine Verallgemeinerung des One-hit-Modells und kann im Prinzip sublinear und superlinear verlaufen. Die Werte für den Gestaltparameter (im Bild mit „b“ bezeichnet) wurden aus den experimentellen Daten berechnet. Theoretisch ist $b=1$ mit dem One-hit-Modell identisch; Werte $b<1$ bedeuten einen superlinearen Kurvenverlauf. Die meisten Kurven verlaufen stark superlinear, auch wenn die höchsten Dosen nicht in die Berechnung einbezogen werden. Auch die Dosis-Häufigkeitsbeziehungen für Amosit, Erionit, Chrysotil und Krokydolith aus den Intraperitonealversuchen von Davis et al. [87] ergaben b-Werte zwischen 0,4 und 0,8. Es waren keine Hinweise auf einen S-förmigen Kurvenverlauf zu erkennen [88]. Vergleichbare Ergebnisse werden von anderen Autoren [87, 89-93] berichtet. Al-sil. $=$ Aluminiumsilikat, Chrys $=$ Chrysotil, $\mathrm{TiO}_{2}=$ Titandioxid, L. = Länge, $D$. = Durchmesser.

selhaft bleibt gleichwohl die geringe Wirkung des Chrysotils „Calidria“. Abb. 2 zeigt, dass auch, bezogen auf die ermittelten Dosen an Fasern länger als $5 \mu \mathrm{m}$ (und Durchmesser kleiner als $2 \mu \mathrm{m}$ ), sehr steile und sehr ähnliche Dosis-Tumorhäufigkeitsbeziehungen sowohl mit Krokydolith als auch mit Chrysotil gefunden wurden. Es gibt dabei keinen Hinweis auf Wirkungsschwellen [78].

\section{In-vitro-Untersuchungen}

In Kulturen von Säugetierzellen zur Prüfung von Gentoxizität in vitro wurde vielfach sowohl mit Amphibolen als auch mit Chrysotil Gentoxizität im Modell nachgewiesen (s. hierzu [60, $103-$ 106]). Nahezu alle Gentoxizitäts- und Zelltransformations-Tests weisen entsprechend den von Asbestfasern in vivo ausgelösten Tumoren positive Ergebnisse auf ( $\bullet$ Tab.4).

\section{Diskussion}

Asbest galt aufgrund seiner technisch hoch erwünschten Eigenschaften bis in die 1970er-Jahre hinein als das Material der 1000 Möglichkeiten. Asbest wurde vor allem als Spritzasbest sowie in der Herstellung bzw. Verwendung von asbesthaltigen Bau- und Dämmmaterialien, Bremsbelägen, Textilien, Gummi- und Kunstharzprodukten eingesetzt [26,45,48,77,107].

Mit einer mittleren Latenzzeit von mehr als 30 Jahren erreichen derzeit nahezu 20 Jahre nach Inkrafttreten des Asbestanwendungsverbots die asbestbedingten Erkrankungen in Deutschland ihren Höhepunkt. Damit geht aktuell eine sozialpolitische, sozio-
Tab.4 In-vitro- und In-vivo-Untersuchungen von Asbest-Faserstäuben (Krokydolith, Chrysotil, Amosit), die alle in-vivo Kanzerogenität aufweisen (detaillierte Darstellung [75].

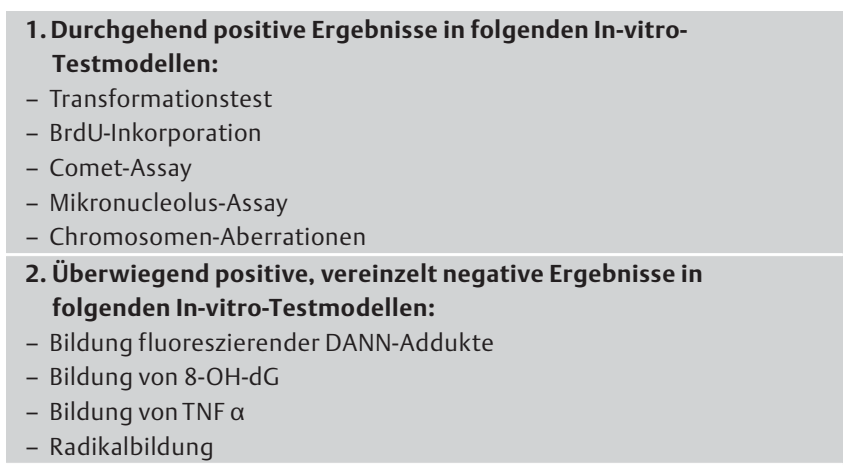

ökonomisch und medizin-ethisch mitgeprägte Diskussion einher, die sich vor allem auf Begutachtensfragen dieser das Berufskrankheitsgeschehen dominierenden Spätfolgen bezieht. Entgegengesetzte Interessenlagen und Sichtweisen kommen dabei zum Tragen [18,108-111].

Im Vordergrund steht derzeit die eingangs erwähnte, für einen Großteil von Erkrankungsfällen seit Jahrzehnten relevante Negierung eines Ursachen-Zusammenhangs des berufsgenossenschaftlichen sog. Deutschen Mesotheliomregisters, wenn im Lungengewebe - auch bei arbeitsmedizinisch und sicherheitstechnisch belegter erheblicher Asbestexposition - keine oder eine vermeintlich zu geringe Konzentration an Asbestkörpern insbesondere bei todbringenden Erkrankungen an Asbestose oder durch Asbest verursachtem Lungenkrebs gefunden werden. Vgl. in diesem Zusammenhang die eindeutigen Statements der internationalen Helsinki-Konferenz in Bezug auf das Fahrerfluchtphänomen des vorwiegend eingesetzten Chrysotil-Asbestes:

"Chrysotile-fibers do not accumulate within lung tissue to the same extent as amphiboles because of faster clearance rates; therefore, occupational histories (fiber-years of exposure) are probably a better indicator of lung cancer risk from chrysotile than a fiber burden analysis is".

"Rare cases of asbestosis in relation to the inhalation of pure chrysotile can occur, with a prolonged interval between the last exposure and the diagnosis and few or no detectable asbestos bodies and a low fiber burden" [112].

Diese Arbeit fasst daher den aktuellen medizinisch wissenschaftlichen Kenntnisstand, insbesondere zu den gesundheitsschädlichen Wirkungen der Chrysotilasbestfasern, welche eine deutlich verkürzte Halbwertzeit im Lungengewebe aufweisen, zusammen. Dabei ist ein Vergleich mit den durch andere Asbestarten bedingten Erkrankungen möglich.

Kein Arbeitsstoff ist bisher hinsichtlich seiner gesundheitsschädlichen Wirkungen so eingehend wie Asbest untersucht worden. Die äußerst umfangreiche Literatur belegt sowohl durch In-vitroals auch durch tierexperimentelle und durch epidemiologische Untersuchungen konsistent die inflammatorischen, toxischen, fibrogenen und kanzerogenen Wirkungen der inhalativ aufgenommenen Asbestfasern. Dabei ist - zusammengefasst - kein prinzipieller Unterschied der gesundheitsschädlichen Wirkungen der verschiedenen Asbestarten festzustellen. So zeigt sich auch, dass alle Asbestfaserarten Lungenfibrosen (Asbestose) und Pleuraplaques induzieren können. Die In-vitro-Gentoxizitätstests an Säugetieren und an deren Zellen weisen weitgehend konsistent positive Befunde auf (s. [71,75-77,28]). Letzteren Ergebnis- 
sen entsprechend waren nach inhalativer oder intraperitonealer Applikation von Asbestfasern die Tumor-induzierenden Wirkungen v.a. in Nagern, z.T. auch in Affen, festzustellen ( Tab.2, - Tab.3; [74-77]).

Die dargestellten Ergebnisse wurden z.T. mit sehr hohen Faserdosen und nach unphysiologischer Applikation (intraperitoneal) erzielt. Hieraus und auch auf Grund von vereinzelten, anatomisch begründbaren Speziesunterschieden leiten sich aber keine abweichenden Interpretationen, sondern vorwiegend Dosis-Wirkungs-Beziehungen der Effekte ab.

Die deutlich längere Beständigkeit von Amphibol-Asbesten im Gewebe gegenüber Chrysotil ist Folge des unterschiedlichen Löslichkeitsverhaltens. Insbesondere aus diesem Unterschied lässt sich eine relativ stärkere pathogene Potenz der ersteren im Vergleich zu Chrysotil ableiten.

Die kürzlich veröffentlichte Metaanalyse der epidemiologischen Studien [31] kommt zu dem Schluss, dass die Beziehungen zwischen Asbestbelastung und Lungenkrebs sehr heterogen sind und Studiendesignmerkmale, wie z.B. die Art der Expositionsbeurteilung, teilweise mit ähnlichen Unterschieden der Lungenkrebspotenziale zusammenhängen wie die Art der Asbestfasern. Unter den Studien zu Chrysotil zeigten die mit höherer Qualität stärkere Lungenkrebspotenziale.

Weitere Forschungsarbeiten sind zur Klärung der z.T. noch offenen Fragen über faserspezifische Wirkungen erforderlich. Die dargelegten epidemiologischen Untersuchungen heben hervor, dass es unabdingbar ist, besonders sorgfältig auf die Qualität der Expositionserfassung in epidemiologischen Studien über berufsund umweltbedingte Risikofaktoren zu achten.

Dabei ist auch die häufig am Arbeitsplatz vorliegende Exposition gegenüber verschiedenen Asbestfasern und anderen Noxen mit gesundheitsschädlichen Wirkungen zu berücksichtigen. Das Argument, die durch Chrysotil-Asbest verursachten Krankheitsbilder seien auf Beimengungen anderer Asbesttypen, v.a. Tremolit (bis 6,9\%; die Amphibolasbestart Tremolit selbst fand keine technische Anwendung) zurückzuführen, lässt sich dadurch entkräften, dass der Tremolit-Anteil nicht mit dem Erkrankungsrisiko korreliert und dass sich auch reines Chrysotil sowohl beim Menschen als auch im Tierversuch als fibrogen und kanzerogen erwies $[22,71,75]$.

Als Fazit für die Begutachtungspraxis sind die hohen Anforderungen an die Arbeitsanamnese hinsichtlich der zu ermittelnden qualitativen und quantitativen arbeitsbedingten kumulativen Belastungen zu beachten. Besondere Zurückhaltung ist ggf. gegenüber vorgelegten und der Arbeitsanamnese widersprechenden Faserkonzentrationen im Gewebe geboten. Hervorzuheben ist die Abgrenzung bzw. Gewichtung von Confoundern, v.a. den Rauchgewohnheiten.

\section{Danksagung}

$\nabla$

Wir danken Herrn Dr. Roller für seine anregenden Kommentierungen zur tierexperimentellen Literatur.

\section{Interessenkonflikt \\ $\nabla$}

Die Autoren geben an, dass kein Interessenkonflikt besteht.

\section{Literatur}

1 Baur X, Clasen M, Fisseler-Eckhoff A et al. Diagnostics and expert opinion of asbestos-induced occupational diseases. Pneumologie 2011; 65: $1-47$

2 Bernstein DM, Rogers $R$, Smith P. The biopersistence of Canadian chrysotile asbestos following inhalation. Inhal Toxicol 2003; 15: $1247-$ 1274

3 Jones JS, Smith PG, Pooley FD et al. The consequences of exposure to asbestos dust in a wartime gas-mask factory. IARC Sci Publ 1980: 637-653

4 McDonald AD, McDonald JC, Pooley FD. Mineral fibre content of lung in mesothelial tumours in North America. Ann Occup Hyg 1982; 26 : 417-422

5 Pooley FD. An examination of the fibrous mineral content of asbestos lung tissue from the Canadian chrysotile mining industry. Environ Res 1976; 12: $281-298$

6 Woitowitz HJ, Manke J, Brückel B et al. Asbestkörperchen als Beweismittel einer beruflichen Gefährdung durch Weißasbest (Chrysotil)? Zbl Arbeitsmed 1986; 36: 354-364

7 Wagner JC, Moncrief CB, Coles $R$ et al. Correlation between fibre content of the lungs and disease in naval dockyard workers. $\mathrm{Br} J$ Ind Med 1986; 43: 391 -395

8 Sebastien P, Begin R. Alveolar clearance of chrysotile in the sheep model - preliminary observations. In: VI International Pneumoconiosis Conference. Bremerhaven: Wirtschaftsverlag NW; 1983: 1010-1020

9 Weller W, Kißler W, Friedrichs $K H$. Fibrogenität ultramikroskopischer Asbest-Fasern. In: VI International Pneumoconiosis Conference. Bremerhaven: Wirtschaftsverlag NW; 1983: 1021-1027

10 Rogers $R$, Smith $P$. The biopersistence of Canadian chrysotile asbestos following inhalation: Final results through 1 year after cessation of exposure. Inhal Toxicol 2005; 17: 1 - 14

11 Rödelsperger K, Woitowitz HJ. Airborne fibre concentrations and lung burden compared to the tumour response in rats and humans exposed to asbestos. Ann Occup Hyg 1995; 39: 715 - 725

12 Heitz P. Neue Definitionen der Minimalasbestose. Dtsch Ärztebl 1997; 94: A975

13 Institut für Pathologie der Ruhr-Universität Bochum. Deutsches Mesotheliomsregister. http://www.bergmannsheil.de/56.0html

14 Müller K-M. Deutsches Mesotheliomregister. BPUVZ; 2005: 691

15 Wiethege T. Differentialdiagnose bösartiger Pleuratumoren - Immunhistochemische und molekularbiologische Untersuchungsbefunde Inaugural-Dissertation. Deutsches MesotheliomregisterUniversität Essen: Zentrum für Pathologie und Rechtsmedizin, Institut für Pathologie; 2000: http://duepublico.uni-duisburg-essen.de/servlets/DerivateServlet/Derivate-10463/Wiethege\%20-\%20Differentialdiagnose\% 20b\%C3\%B6sartiger\%20Pleuratumoren.pdf

16 PohlmannJ. Immunhistochemische Differentialdiagnose von kleinzelligen Lungenkarzinomen und epitheloiden Mesotheliomen. Inaugural-Dissertation. Hohe Medizinische Fakultät der Ruhr-UniversitätBochum; 2007: http://www-brs.ub.ruhr-uni-bochum.de/netahtml/ HSS/Diss/PohlmannJanine/diss.pdf

17 Kanzlei Battenstein \& Battenstein. Mesotheliomregister. 2011: http:// www.battenstein.com/blog/tag/mesotheliomregister/

18 Clasen M. Stellungnahme des Bundesverbandes der Asbestose Selbsthilfegruppen. In: Falkensteiner Tage 2010 Kolloquium zur Begutachtung asbestbedingter Berufskrankheiten. Berlin: DGUV; 2011: 129133

19 Deutsche Gesetzliche Unfallversicherung. Spitzenverband der landwirtschaftlichen Sozialversicherung. Empfehlung für die Begutachtung asbestbedingter Berufskrankheiten - Falkensteiner Empfehlung. Berlin: DGUV; 2011

20 Neumann $V$, Theile A, Loseke S et al. Neue Aspekte zur Pathogenese der Asbestose. Arbeitsmed Sozialmed Umweltmed 2011; 46: 569-579

21 McDonald JC, McDonald AD. Chrysotile, tremolite and carcinogenicity. Ann Occup Hyg 1997; 41: 699-705

22 Stayner LT, Dankovic DA, Lemen RA. Occupational exposure to chrysotile asbestos and cancer risk: a review of the amphibole hypothesis. Am J Public Health 1996; 86: 179-186

23 Editorial. Asbestos scandal. Nature 2010; 468: 868

24 Canadian Environmental Law Association. Stephen Harper's killer legacy. 2010: http://www.cela.ca/newsevents/advertisement/stephen-harpers-killer-legacy

25 Schneider J, Rödelsperger K. Staub und Staubinhaltsstoffe/Asbest. In: Wichmann HE, Schlipköter HW, Fülgraff G eds. Handbuch der Um- 
weltmedizin Toxikologie, Epidemiologie, Hygiene, Belastungen, Wirkungen, Diagnostik, Prophylaxe. Landsberg: ecomed; 1998: 2-37

26 Senatskommission zur Prüfung gesundheitsschädlicher Arbeitsstoffe der DFG. Asbest-Feinstaub und asbesthaltiger Feinstaub. Zur Frage von Unterschieden in der gesundheitsschädlichen Wirkung der Asbestarten Chrysotil, Amosit und Krokydolith Nachtrag 1981. In: Hartwig A ed. Gesundheitsschädliche Arbeitsstoffe Toxikologisch-arbeitsmedizinische Begründungen von MAK-Werten (Maximale Arbeitsplatzkonzentrationen). Weinheim: WILEY-VCH; 1981

27 IARC. Asbestos (chrysotile, amosite, crocidolite, tremolite, actinolite, and anthophyllite). In: IARC Working group on the evaluation of carcinogenic risks to humans ed. A review of human carcinogens. Part C: Arsenic, metals, fibres, and dusts. Lyon: IARC; 2009: 219-310 http:// monographs.iarc.fr/ENG/Monographs/vol100C/mono100C-11.pdf

28 WHO, IARC. Asbestos (Chrysotile, Amosite, Crocidolite, Tremolite, Actinolite and Anthophyllite). A Review of Human Carcinogens: Arsenic, Metals, Fibres, and Dusts Lyon: WHO, IARC; 2012

29 WHO, IARC. eds. Overall evaluations of carcinogenicity: An updating of IARC Monographs volumes 1 to 42. Supplement 7. Lyon: IARC; 1987: http://monographs.iarc.fr/ENG/Monographs/suppl7/suppl7. pdf

30 Berman DW, Crump KS. A meta-analysis of asbestos-related cancer risk that addresses fiber size and mineral type. Crit Rev Toxicol 2008; 38: $49-73$

31 Lenters $V$, Vermeulen $R$, Dogger $S$ et al. A Meta-Analysis of Asbestos and Lung Cancer: Is Better Quality Exposure Assessment Associated with Steeper Slopes of the Exposure-Response Relationships? Environ Health Perspect 2011

32 Deng $Q$ Wang $X$, Wang $M$ et al. Exposure-response relationship between chrysotile exposure and mortality from lung cancer and asbestosis. Occup Environ Med 2012; 69: 81 - 86

33 Wang X, Yano E, Qiu $H$ et al. A 37-year observation of mortality in Chinese chrysotile asbestos workers. Thorax 2012; 67: 106-110

34 Wang $X$, Lin S, Yano E et al. Mortality in a Chinese chrysotile miner cohort. Int Arch Occup Environ Health 2011

35 Elliott L, Loomis D, Dement J et al. Lung cancer mortality in North Carolina and South Carolina chrysotile asbestos textile workers. Occup Environ Med 2012; 69: 385-390

36 Hein MJ, Stayner LT, Lehman E et al. Follow-up study of chrysotile textile workers: cohort mortality and exposure-response. Occup Environ Med 2007; 64: 616-625

37 Loomis D, Dement JM, Wolf SH et al. Lung cancer mortality and fibre exposures among North Carolina asbestos textile workers. Occup Environ Med 2009; 66: 535-542

38 Liddell FD, McDonald AD, McDonald JC. The 1891-1920 birth cohort of Quebec chrysotile miners and millers: development from 1904 and mortality to 1992. Ann Occup Hyg 1997; 41: 13-36

39 Pira E, Pelucchi C, Piolatto PG et al. Mortality from cancer and other causes in the Balangero cohort of chrysotile asbestos miners. Occup Environ Med 2009; 66: 805-809

$40 \mathrm{McDonald} A D$, Fry JS, Woolley AJ et al. Dust exposure and mortality in an American chrysotile asbestos friction products plant. $\mathrm{Br} \mathrm{J}$ Ind Med 1984; 41: $151-157$

41 Sullivan PA. Vermiculite, respiratory disease, and asbestos exposure in Libby, Montana: update of a cohort mortality study. Environ Health Perspect 2007; 115: 579-585

42 Berry G, de Klerk NH, Reid A et al. Malignant pleural and peritoneal mesotheliomas in former miners and millers of crocidolite at Wittenoom, Western Australia. Occup Environ Med 2004; 61: 14

43 Levin JL, McLarty JW, Hurst GA et al. Tyler asbestos workers: mortality experience in a cohort exposed to amosite. Occup Environ Med 1998; 55: $155-160$

44 Seidman H, Selikoff IJ, Gelb SK. Mortality experience of amosite asbestos factory workers: dose-response relationships 5 to 40 years after onset of short-term work exposure. Am J Ind Med 1986; 10: 479-514

45 IPCS. ed. Asbestos and other natural mineral fibres. Geneva: WHO; 1986

46 WHO. Air quality guidelines for Europe. 2nd: ed. Copenhagen: WHO; 2000

47 WHO, IARC. Some inorganic and organometallic compounds. Summary of data reported and evaluation. IARC Monographs on the Evaluation of Carcinogenic Risks to Humans Lyon: IARC; 1998; 2: http:// monographs.iarc.fr/ENG/Monographs/vol2/volume2.pdf

48 IARC. ed. Asbestos. Summary of data reported and evaluation Lyon: 1998
49 Schneider J, Woitowitz HJ. Pleuramesotheliom. In: Wichmann HE, Schlipköter HW, Füllgraff G eds. Handbuch der Umweltmedizin Toxikologie, Epidemiologie, Hygiene, Belastungen, Wirkungen, Diagnostik, Prophylaxe. Landsberg: ecomed; 1995: 1-20

50 Piolatto G, Negri E, La Vecchia C et al. An update of cancer mortality among chrysotile asbestos miners in Balangero, northern Italy. Br J Ind Med 1990; 47: 810-814

51 Hodgson JT, Darnton A. Mesothelioma risk from chrysotile. Occup Environ Med 2010; 67: 432

52 Kanarek MS. Mesothelioma from chrysotile asbestos: update. Ann Epidemiol 2011; 21: 688-697

53 Dumortier P, Rey F, Viallat JR et al. Chrysotile and tremolite asbestos fibres in the lungs and parietal pleura of Corsican goats. Occup Environ Med 2002; 59: 643-646

54 Mitchev K, Dumortier P, De Vuyst P. 'Black Spots' and hyaline pleura plaques on the parietal pleura of 150 urban necropsy cases. Am J Surg Pathol 2002; 26: $1198-1206$

55 Bundesministerium für Arbeit und Sozialordnung. Merkblatt zur BK Nr. 4104: Lungenkrebs oder Kehlkopfkrebs in Verbindung mit Asbeststaublungenerkrankung (Asbestose), in Verbindung mit durch Asbeststaub verursachter Erkrankung der Pleura oder bei Nachweis der Einwirkung einer kumulativen Asbestfaserstaub-Dosis am $\mathrm{Ar}-$ beitsplatz von mindestens 25 Faserjahren $(25 \times 106$ [(Fasern $/ \mathrm{m} 3) \times$ Jahre]). Bek. des BMA v. 1.12.1997- IVa 4-45206. BArbBl; 1997: 32 35

56 Pira E, Pelucchi C, Buffoni $L$ et al. Cancer mortality in a cohort of asbestos textile workers. Br J Cancer 2005; 92: 580 - 586

57 Institute of Medicine of the National Academies. ed. Asbestos. Selected cancers. Committee on Asbestos: Selected Health Effects. Board on Population Health and Public Health Practices. Washington, D. C: The National Academies; 2006

58 Straif K, Benbrahim-Tallaa L, Baan $R$ et al. A review of human carcinogens - part C: metals, arsenic, dusts, and fibres. Lancet Oncol 2009; 10: $453-454$

59 Camargo MC, Stayner LT, Straif $K$ et al. Occupational exposure to asbestos and ovarian cancer: a meta-analysis. Environ Health Perspect 2011; 119: $1211-1217$

60 Mossman BT, Bignon J. Asbestos: scientific developments and implications for public policy. Science 1990; 247: 4940294-301

61 U.S. Department of Health and Human Services. Toxicological profile for asbestos. Atlanta, Georgia: U.S. Department of Health and Human Services Public Health Service Agency for Toxic Substances and Disease Registry; 2001: 441

62 Musk AW, de Klerk NH, Reid A et al. Mortality of former crocidolite (blue asbestos) miners and millers at Wittenoom. Occup Environ Med 2008; 65: 541 - 543

63 Woitowitz H-J, Schäcke G, Woitowitz R. Zu den Auswirkungen von Pleuraverkalkungen bei Chrysotil-Asbestarbeitern auf die Lungenfunktion. Med Welt 1971; 22 : 931-935

64 Broaddus VC, Everitt JI, Black B et al. Non-neoplastic and neoplastic pleural endpoints following fiber exposure. J Toxicol Environ Health B Crit Rev 2011: 14: 153-178

65 Ameille J, Rosenberg $N$, Matrat $M$ et al. Asbestos-related diseases in automobile mechanics. Ann Occup Hyg 2012; 56: 55-60

66 Liddell $D$. Cancer mortality in chrysotile mining and milling: exposure-response. Ann Occup Hyg 1994; 38: 519-523 412

67 Becklake MR, Liddell FD, Manfreda J et al. Radiological changes after withdrawal from asbestos exposure. Br J Ind Med 1979; 36: 23-28

68 Rubino GF, Newhouse M, Murray R et al. Radiologic changes after cessation of exposure among chrysotile asbestos miners in Italy. Ann N Y Acad Sci 1979; 330: 157-161

69 Berry G, Gilson JC, Holmes $S$ et al. Asbestosis: a study of dose-response relationships in an asbestos textile factory. $\mathrm{Br} \mathrm{J}$ Ind Med 1979; 36: $98-112$

70 Viallat JR, Boutin C, Pietri JF et al. Late progression of radiographic changes in Canari chrysotile mine and mill exworkers. Arch Environ Health 1983; 38: 54-58

71 WHO. ed. International Programme on Chemical Safety (IPCS). Chrysotile asbestos Geneva: 1998

72 Baur X, Wilken D. Effect of asbestos fibre dust exposures on lung function-a systematic review. Pneumologie 2010; 64: 81 - 110

73 Wilken D, Velasco Garrido M, Manuwald $U$ et al. Lung function in asbestos-exposed workers, a systematic review and meta-analysis. J Occup Med Toxicol 2011; 6: 21 
74 Risk Assessment Information System (RAIS). ed. Toxicity summary for asbestos. Oak Ridge: Chemical Hazard Evaluation and Communication Group, Biomedical and Environmental Information Analysis Section. Health and Safety Research Division; 1995

75 Roller $M$. Bedeutung von In-vitro-Methoden zur Beurteilung der chronischen Toxizität und Karzinogenität von Nanomaterialien, Feinstäuben und Fasern. Dortmund, Berlin: BAuA; 2011: 1 - 365

76 Roller M, Akkan Z, Hassauer M, Kalberlah F. Risikoextrapolation vom Versuchstier auf den Menschen bei Kanzerogenen - Forschung $\mathrm{Fb}$ 1078. Bremerhaven: BAuA. Wirtschaftsverlag NW; 2006

77 WHO. ed. International Programme on Chemical Safety (IPCS). Asbestos and other natural mineral fibres Geneva: 1986

78 Pott F, Roller M, Althoff GH et al. Beurteilung der Kanzerogenität von inhalierbaren Fasern. In: Kommission Reinhaltung der Luft im VDI und DIN Faserförmige Stäube Vorschriften Wirkungen Messung Minderung Tagung Fulda, 7-9 September 1993. Düsseldorf: VDI-Verlag; 1993: $17-77$

79 Pott F, Roller M, Ziem $U$ et al. Carcinogenicity studies on natural and man-made fibres with the intraperitoneal test in rats. In: Bignon J, Peto J, Saracci R eds. Non-occupational Exposure to Mineral Fibres. Lyon: International Agency for Research on Cancer; 1989: 173-179

80 Davis JM, Jones AD. Comparisons of the pathogenicity of long and short fibres of chrysotile asbestos in rats. Br J Exp Pathol 1988; 69: $717-737$

81 Davis JM, Beckett ST, Bolton RE et al. Mass and number of fibres in the pathogenesis of asbestos-related lung disease in rats. $\mathrm{Br} \mathrm{J}$ Cancer 1978; 37: $673-688$

82 Davis JM, Beckett ST, Bolton RE et al. The effects of intermittent high asbestos exposure (peak dose levels) on the lungs of rats. $\mathrm{Br} \mathrm{J}$ Exp Pathol 1980; 61: 272-280

83 Davis JM, Addison J, Bolton RE et al. The pathogenicity of long versus short fibre samples of amosite asbestos administered to rats by inhalation and intraperitoneal injection. Br J Exp Pathol 1986; 67: 415 430

84 Davis JM, Bolton RE, Douglas AN et al. Effects of electrostatic charge on the pathogenicity of chrysotile asbestos. Br J Ind Med 1988; 45: 292 299

85 Pott F, Ziem U, Reiffer FJ et al. Carcinogenicity studies on fibres, metal compounds, and some other dusts in rats. Exp Pathol 1987; 32: 129152

86 Pott F, Dungworth DL, Heinrich $U$ et al. Lung tumours in rats after intratracheal instillation of dusts. Ann Occup Hyg 1994; 38: 357 - 363

87 Davis JM, Bolton RE, Miller BG et al. Mesothelioma dose response following intraperitoneal injection of mineral fibres. Int J Exp Pathol 1991; 72: $263-274$

88 Pott F, Roller M. Die krebserzeigende Wirkung von Fasern unter besonderer Berücksichtigung der Inhalationsversuche. Dortmund: Bundesanstalt für Arbeitsschutz; 1993

89 Davis JM, Addison J, Bolton RE et al. Inhalation studies on the effects of tremolite and brucite dust in rats. Carcinogenesis 1985; 6: 667-674

90 Davis JM, Addison J, Bolton RE et al. Inhalation and injection studies in rats using dust samples from chrysotile asbestos prepared by a wet dispersion process. Br J Exp Pathol 1986; 67: 113-129

91 Davis JM, Beckett ST, Bolton RE et al. A comparison of the pathological effects in rats of the UICC reference samples of amosite and chrysotile with those of amosite and chrysotile collected from the factory environment. IARC Sci Publ 1980: 285 - 292

92 Wagner JC, Berry G, Skidmore JW et al. The effects of the inhalation of asbestos in rats. Br J Cancer 1974; 29: 252 - 269

93 Wagner JC, Griffiths DM, Hill RJ. The effect of fibre size on the in vivo activity of UICC crocidolite. Br J Cancer 1984; 49: $453-458$

94 Pott F, Roller M, Althoff GH. Krebsrisiko durch Fasern - ein zusammenfassender Vergleich von epidemiologischen und tierexperimentellen
Daten. In: Gesellschaft zur Förderung der Lufthygiene und Silikoseforschung e.V Umwelthygiene. 27. Jahresbericht 1994/95. Düsseldorf: Medizinisches Institut für Umwelthygiene; 1995: 133 - 200

95 Mast RW, McConnell EE, Anderson R et al. Studies on the chronic toxicity (inhalation) of four types of refractory ceramic fiber in male Fischer 344 rats. Inhal Toxicol 1995; 7: 425-467

96 McConnell EK, Kamstrup O, Musselman $R$ et al. Chronic inhalation study of size-separated rock and slag wool insulation fibers in Fischer 344/N rats. Inhal Toxicol 1994; 6: $571-614$

97 Pott F, Friedrichs $K H$, Huth F. Results of animal experiments concerning the carcinogenic effect of fibrous dusts and their interpretation with regard to the carcinogenesis in humans. Zentralbl Bakteriol Orig B 1976; 162: 467 - 505

98 Pott F, Schlipköter HW, Ziem U et al. New results from implantation experiments with mineral fibres. In: WHO Biological effects of manmade mineral fibres Proceedings of a WHO/IARC Conference 1984. Copenhagen: Regional Office for Europe; 1984: 286-302

99 Pott F, Roller M, Ziem $U$ et al. Carcinogenicity studies on natural and man-made fibres with the intraperitoneal test in rats. In: Bignon J, Peto J, Saracci R eds. Non-occupational exposure to mineral fibres. Lyon: International Agency for Research on Cancer; 1989: 173-179

100 Pott F, Bellmann B, Muhle $H$ et al. Intraperitoneal injection studies for the evaluation of the carcinogenicity of fibrous phyllosilicates. In: Bignon J ed. Health related effects of phyllosilicates (NATO ASI Series). Berlin: Springer; 1990: 319-329

101 Roller M, Pott F, Kamino K et al. Results of current intraperitoneal carcinogenicity studies with mineral and vitreous fibres. Exp Toxicol Pathol 1996; 48: 3-12

102 Roller M, Pott F, Kamino K et al. Dose-response relationship of fibrous dusts in intraperitoneal studies. Environ Health Perspect 1997; 105: $1253-1256$

103 Bhattacharya K, Dopp E, Kakkar P et al. Biomarkers in risk assessment of asbestos exposure. Mutat Res 2005; 579: 6-21

104 Poser I, Rahman $Q$ Lohani $M$ et al. Modulation of genotoxic effects in asbestos-exposed primary human mesothelial cells by radical scavengers, metal chelators and a glutathione precursor. Mutat Res 2004: 559: 19-27

105 Schins RP. Mechanisms of genotoxicity of particles and fibers. Inhal Toxicol 2002; 14: $57-78$

106 Speit $G$. Appropriate in vitro test conditions for genotoxicity testing of fibers. Inhal Toxicol 2002; 14: 79-90

107 Baur X, Schneider J, Woitowitz H-J. Diagnostik und Begutachtung asbestbedingter Berufskrankheiten. Dtsch Med Wochenschr 2011; 136: 2319-2324

108 Baur X, Wilken D. Antwort auf Leserbriefe zu X. Baur, D. Wilken. Auswirkungen der Asbestfaserstaub-Exposition auf die Lungenfunktion - ein systematisches Review.. Pneumologie 2010; 64: 81-110 Pneumologie 2010; 64: 436-441

109 Müsken H. Leserbrief zu X. Baur, D. Wilken. Auswirkungen der Asbestfaserstaub-Exposition auf die Lungenfunktion - ein systematisches Review.. Pneumologie 2010; 64: 81-110 Pneumologie 2010; 64: 433

110 Neumann V, Tannapfel A. Leserbrief zu X. Baur, D. Wilken. Auswirkungen der Asbestfaserstaub-Exposition auf die Lungenfunktion - ein systematisches Review.. Pneumologie 2010; 64: 81-110 Pneumologie 2010; 64: 430-432

111 Beyer V. Stellungnahme des Deutschen Gewerkschaftsbundes (DGB). Falkensteiner Tage 2010 Kolloquium zur Begutachtung asbestbedingter Berufskrankheiten Berlin: DGUV; 2011: 125-127

112 Henderson D, Rantanen J, Barnhart $S$ et al. Asbestos, asbestosis and cancer: the Helsinki criteria for diagnosis and attribution. Scand J Work Environ Health 1997; 23: 311 -316 